\title{
Body Discomfort Due to Musculoskeletal Disorders among Dental Practitioners in Primary Healthcare Center in Bandung City
}

\author{
Nur Ismah Gitasari ${ }^{1}$, Sri Tjahajawati $^{2 *}$, Rosilawati Wihardja ${ }^{2}$ \\ ${ }^{1}$ Faculty of Dentistry, Padjadjaran University, Bandung 40134, Indonesia \\ ${ }^{2}$ Department of Oral Biology, Faculty of Dentistry, Padjadjaran University, Bandung 40134, \\ Indonesia \\ *Email: sri.tjahajawati@fkg.unpad.ac.id
}

\begin{abstract}
Musculoskeletal disorders (MSD) are one of the main occupational health hazards affecting dental practitioners and result in discomfort, pain, and illness that can result in disruption or impairment of dental practice. The objective of this study is to discover the description of MSD among dental practitioners at the Primary Healthcare Center (Puskesmas) in Bandung City. This descriptive study was conducted using a cross-sectional survey. The study sample consisted of 27 dentists (23 females and 4 males) working at the Primary Healthcare Center in Bandung City who were asked to fill out the Cornell Musculoskeletal Discomfort Questionnaire. The study showed that the type of discomfort most commonly experienced was sore muscles $(57.5 \%)$, with the highest number of complaints in the axial region affecting the lower back $(41.4 \%)$, in the upper limb region specifically the right shoulder $(8.3 \%)$, and in lower limb region specifically the right knee $(0.53 \%)$. It was concluded that dentists at the Primary Healthcare Center in Bandung experienced sore muscles due to MSD and most cases of MSD involved the lower back, the right shoulder, and the right knee.
\end{abstract}

Keywords: body discomfort, cornell musculoskeletal discomfort questionnaire (CMDQ), musculoskeletal disorders (MSD)

\section{Introduction}

Dental work cannot be categorized as an undemanding job, because dentists must perform repetitive and static movements for extended periods of time, involving multiple parts of the body, and requiring high levels of concentration [1, 2]. Dentists have high rates of musculoskeletal disorders (MSD) due in part to contributing factors including holding static posture for long periods, performing repetitive upper limb motions, doing heavy lifting, using high-vibration instruments, working in limited and confined areas, having insufficient lighting, experiencing psychological disturbances, and advancing age [3-5].

The World Health Organization defines MSD as "a disorder of the muscles, tendons, joints, intervertebral discs, peripheral nerves, and vascular system, not directly resulting from an acute or instantaneous event but installing gradually and chronically" [6]. An MSD is one of the major occupational health problems faced by the dentists since it often causes discomfort, minor aches and pains in many parts of the body. In some cases, the severity of the condition may result in both 
significant social and economic consequences, such as reduced quality of dental care provided, inability to work, and even leaving the profession $[1,7]$.

Musculoskeletal disorders in dentists have been the subject of numerous studies worldwide, and the overall prevalence of musculoskeletal disorders in dentists ranges from 66 to 93 percent [7-10]. Various studies have shown that when MSD is found in dentists, it is located in specific regions such as the neck $[5,10,11]$ and the lower back [12-14]. The purpose of this study is to describe the most common types and locations of MSD in dentists working at the Public Healthcare Center in Bandung City.

\section{Materials and Methods}

A cross-sectional descriptive study was conducted in 2016. Twenty-seven dentists (23 females and 4 males) participated in this study. The participants were asked to complete the Cornell Musculoskeletal Discomfort Questionnaires (CMDQ). Questionnaires were used to collect the required data related to the prevalence of MSDs. The symptoms of MSDs in this study were defined as discomfort, pain, fatigue, limited range of motion, and loss of motor control in the neck, shoulders, and wrists, hand, and both upper and lower back.

The CMDQ included a diagram of the human body (viewed from the back, divided into the twelve anatomical areas) to help participants identify areas of the body. The questions targeted three parameters: frequency, discomfort level, and interference (see Fig. 1). In this study, the total score is analyzed by weighing the rating scores, thus aiding the identification of the most serious problems. The scoring was as follows: never $=0,1-2$ times/week $=1,3-4$ times/week $=2$, every day $=3$, and several times every day $=4$. By multiplying each of the above frequency scores $(0,1,2,3,4)$ by the discomfort score $(1,2,3)$, by the interference score $(0$, $1,2)$, yielded a product equaling the total score. Fig 1 illustrates the score associated with each body part gathered from the questionnaire [15].

\section{Results}

Eighty-eight percent of subjects experienced discomfort because of MSD in at least one body region. The most common type of discomfort experienced by respondents were sore muscles $(57.2 \%)$.

Both the score and percentage obtained as a result of processing the data from the questionnaire is divided into three body regions; axial region, upper extremity region, and lower extremity region. The lower back had the highest percentage of MSD in the axial body region (41.4\%), followed by the neck (16.6\%). The upper back and pelvis have a small percentage of MSD when compared with areas of both the neck and lower back.

In the upper extremity region, the right shoulder had the highest percentage $(8.3 \%)$ of MSD compared with other upper extremities and the right knee had the highest percentage $(0.53 \%)$ of MSD in the lower extremity area. 


\section{Discussion}

From 21 primary health care centers (Puskesmas) in Bandung city, 27 subjects were asked to fill out the questionnaire. The objective of this study was to evaluate the type of MSD-related body discomfort most often experienced by dentists and the location of MSD in three separate body regions; axial, upper extremity and lower extremity. Based on this research, the prevalence of MSD among dentists at the Primary Healthcare Center in Bandung was $88 \%$, and sore muscles were significantly more prevalent than any other type of body discomfort experienced by respondents.

Every day, dentists typically work in a static and rigid position for long periods of time that requires continuous muscle contraction. These conditions will lead to consequences of discomfort such as aches, pains, or cramps leading to MSD [16]. The process of muscle soreness begins with the presence of tension or excessive pull on the muscle's functional unit called a sarcomere. The excessive tension on the sarcomere further triggers the breakdown of the sarcomere, causing intracellular calcium accumulation. This process will be followed by an inflammatory response, with the recruitment of inflammatory cells and cytokines that will activate specific pain receptors, called nociceptors, which are responsible for the perception of muscle pain and soreness [17, 18].

Pain in the lower back, as shown in this study was significantly more prevalent than in any other parts of the axial region, and this finding was consistent with Dantas et al. In addition, this finding was consistent with many other studies [13, 14]. The main risk factor for lower back pain associated with dental work is the poor and unsupported sitting position. Dentists tend to bend their backs and bow their necks to maximize the view and access to the patient's mouth. This position will cause disturbances in various parts of the body, especially on the spinal disc that causes lower back pain $[19,20]$. Spinal disks provide movement between the vertebrae for spinal flexibility, and both sustain and transmit the pressure between them. Disks are composed of a tough, outer layer (the annulus fibrosus) and a gellike mass in the center (the nucleus pulposus). The pressure on lumbar disks in the seated position is more than $50 \%$ than that when standing. Bending (forward flexion) and twisting (rotation) positions of the back will increase the pressure on the lumbar disks up to $200 \%$. This type of pressure on the disk can lead to a bulge or herniation of the spinal disk, causing compression on a spinal nerve, leading to lower back, hip, or leg pains [16, 19].

In the upper extremity, the right shoulder has the highest score and the percentage of MSD than any other parts of the upper extremity. These findings for shoulder complaints in this study were supported by Feng et al. and Rafie et al. who showed that shoulder pain had the highest percentage of MSD in the upper extremity region. Shoulder complaints result from dentist's work positions that require the dentist to lift or elevate his or her shoulder (with flexion or abduction more than $30^{\circ}$ ), thereby causing excessive pressure on the shoulders [5, 8]. Most injuries and shoulder pain occur because of interference with both ligaments and tendons, not bones. Handling small instruments requires awkward arm and shoulder positions to reach specific regions within the mouth, which inevitably increases the load on the shoulder. Pain in the shoulder includes tendinitis and injury to 
the muscles, tendons, and ligaments. The tendon on the shoulder is very vulnerable to experience inflammation caused by the unstable shoulder joint because of the considerably larger size of the head of the humerus articulating with the glenoid fossa $[8,21]$.

Complaints involving the knee were more prevalent than any other part of the lower extremity region. In addition, the prevalence of MSD symptoms in the knee is higher when compared with other regions in the lower limb. Knee disorders are associated with bursitis, a condition that develops in response to friction or direct pressure on the knee [22]. Bursitis in dentists occurs due to the unsuitable sitting position of dentists since the angle formed at the knee is less than $90^{\circ}$, causing pressure on the bursa. Because all respondents were right-handed, their sitting positions tilted to the right side to maximize their views into their patient's mouths. This type of positional shift also causes greater pressure for the right knee when compared with the left knee, so symptoms of MSD will be more common in the right knee.

\section{Conclusion}

In conclusion, pain, injury, or MSD are consequences of the working process in dentistry and represent important causes of morbidity and work inefficiency. The limitation of this study lies in the lack of direct observation of the respondents, how they work, and the application of dental ergonomics in the working area. Further research emphasizing direct observation is required to address risk factors of MSD in working areas accurately.

\section{Acknowledgments}

We would like to thank the dean of the Faculty of Dentistry Padjadjaran University, and to the head of Bandung City Health Department who has given permission and support for this research. Also to dentists at Public Healthcare Center in Bandung City who kindly collaborated with this research.

\section{References}

1. Bedi HS, Moon NJ, Bhatia V, Sidhu GK, Khan N. Evaluation of musculoskeletal disorders in dentists and application of DMAIC technique to improve the ergonomics at dental clinics and meta-analysis of literature. J Clin Diagno Res. 2015;9(6):ZC01.

2. Anghel M, Argesanu V, Talpos-Niculescu C, Lungeanu D. Musculoskeletal disorders (MSDs)-consequences of prolonged static postures. J Exp Med Surgic Res. 2007;4:16772.

3. Gupta A, Manohar Bhat TM, Bansal N, Gupta G. Ergonomics in dentistry. Int J Clin Ped Dent. 2014;7(1):30.

4. Nordander C, Hansson GA, Ohlsson K, Arvidsson I, Balogh I, Strömberg U, Rittner R, Skerfving S. Exposure-response relationships for work-related neck and shoulder musculoskeletal disorders-Analyses of pooled uniform data sets. Appl Ergon. 2016;55:7084 .

5. Rafie F, Zamani Jam A, Shahravan A, Raoof M, Eskandarizadeh A. Prevalence of upper extremity musculoskeletal disorders in dentists: symptoms and risk factors. J Environ Public Health. 2015;2015. 
6. Singla R, Gupta H, Kaur I, Singla K, Singh J, Aggarwal S. Dent J Adv Studies. 2015;3:66-70.

7. Lin TH, Liu YC, Hsieh TY, Hsiao FY, Lai YC, Chang CS. Prevalence of and risk factors for musculoskeletal complaints among Taiwanese dentists. J Dent Sci. 2012;7(1):65-71.

8. Feng B, Liang Q, Wang Y, Andersen LL, Szeto G. Prevalence of work-related musculoskeletal symptoms of the neck and upper extremity among dentists in China. BMJ Open. 2014 Dec 1;4(12):e006451.

9. Hodacova L, Sustova Z, Cermakova E, Kapitan M, Smejkalova J. Self-reported risk factors related to the most frequent musculoskeletal complaints among Czech dentists. Industrial Health. 2015;53(1):48-55.

10. Decharat S, Phethuayluk P, Maneelok S. Prevalence of musculoskeletal symptoms among dental health workers, Southern Thailand. Adv Prev Med. 2016;2016.

11. Fish DR, Morris-Allen DM. Musculoskeletal disorders in dentists. N Y State Dent J. 1998 Apr;64(4):44-8.

12. Dantas FF, de Lima KC. The relationship between physical load and musculoskeletal complaints among Brazilian dentists. Appl Ergon. 2015 Mar 31;47:93-8.

13. Al-Mohrej OA, AlShaalan NS, Al-Bani WM, Masuadi EM, Almodaimegh HS. Prevalence of musculoskeletal pain of the neck, upper extremities and lower back among dental practitioners working in Riyadh, Saudi Arabia: a cross-sectional study. BMJ Open. 2016;6(6):e011100.

14. Park HS, Kim J, Roh HL, Namkoong S. Analysis of the risk factors of musculoskeletal disease among dentists induced by work posture. J Phys Ther Sci. 2015;27(12):3651-4.

15. Jansen K, Luik M, Reinvee M, Viljasoo V, Ereline J, Gapeyeva H, Pääsuke M. Musculoskeletal discomfort in production assembly workers. Acta Kinesiologiae Universitatis Tartuensis. 2012;18:102-10.

16. Valachi B, Valachi K. Mechanisms leading to musculoskeletal disorders in dentistry. J Am Dent Assoc. 2003 Oct 1;134(10):1344-50.

17. Lewis PB, Ruby D, Bush-Joseph CA. Muscle soreness and delayed-onset muscle soreness. Clin Sport Med. 2012 Apr 1;31(2):255-62.

18. Mense S. Muscle pain: mechanisms and clinical significance. Deutsches Ärzteblatt Int. 2008 Mar;105(12):214.

19. Occupational Health for Ontario Workers. Ergonomics and Dental Work. [Last accessed on 2014 Sep 11]. Available from: http://www.ohcow.on.ca/uploads/

20. British Dental Association. Occupational back pain - Factsheet. 2010.

21. Hayes MJ, Cockrell D, Smith DR. A systematic review of musculoskeletal disorders among dental professionals. Int J Dent Hyg. 2009 Aug 1;7(3):159-65.

22. Hill H. Lower limb MSD. Health and Safety Executive. 2009. 